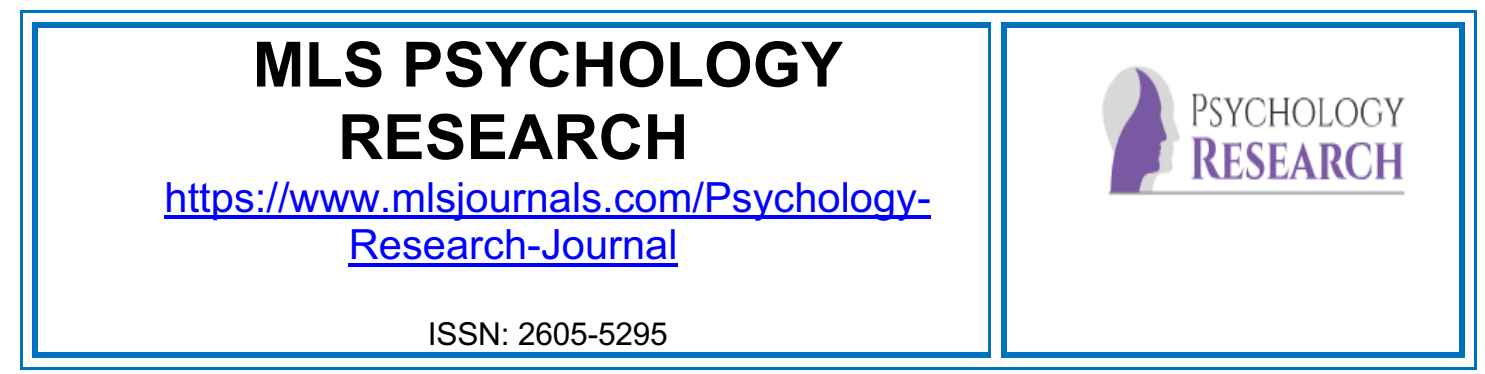

Cómo citar este artículo:

Álvarez, N. (2020). .Ausencia de protocolos de prevención de trata humana para menores de edad en Puerto Rico. MLS Psychology Research 3 (1), 65-78. doi: 10.33000/mlspr.v2i2.463

\title{
AUSENCIA DE PROTOCOLOS DE PREVENCION DE TRATA HUMANA PARA MENORES DE EDAD EN PUERTO RICO
}

\author{
Nydia I Alvarez \\ Funiber - Fundación Universitaria Iberoamericana (Puerto Rico) \\ nydia.alvarez@gmail.com·https://orcid.org/0000-0002-3348-1416
}

\begin{abstract}
Resumen: La trata humana, es un fenómeno que crece en las entrañas de muchos países del mundo. Puerto Rico, no es la excepción a esta situación. En la investigación que se presenta en este artículo, se analizan las repercusiones que ha tenido la ausencia de protocolos de trata humana para menores de edad, en la lucha contra este fenómeno en Puerto Rico. Para lograr ese propósito, se realizó una investigación de enfoque mixto y diseño exploratorio. Respecto a las hipótesis del estudio, a través de estas, se intentó probar que las efectos negativos que acarrea la ausencia de protocolos, en la lucha contra este fenómeno, disminuirían con la presencia de protocolos de trata humana para menores en Puerto Rico. También, se auscultó si la identificación e inclusión de los factores correctos, en un protocolo para menores, podría mejorar las estrategias de detección de casos. Se utilizó la percepción y conocimiento de expertos que ofrecen servicios a la población de menores, en algunas agencias públicas de Puerto Rico y en algunas organizaciones no gubernamentales (ONG). La muestra seleccionada, fue no aleatoria y por disponibilidad. La técnica utilizada para obtener la información, fue la entrevista por medio de un cuestionario. El cuestionario, se redactó utilizando una escala Likert, además, se realizaron preguntas abiertas. Se cumplió con los objetivos principales de crear un prototipo de plan de prevención juvenil de trata huma e identificar los factores que debe incluir un protocolo de prevención y protección de la trata para menores en Puerto Rico.
\end{abstract}

Palabras clave: trata humana, derechos humanos, menores, esclavitud y explotación.

\section{ABSENCE OF PROTOCOLS FOR PEVENTION THE HUMAN TRAFFICKING OF MINORS IN PUERTO RICO}

Summary: Human trafficking is a phenomenon that grows in the bowels of many countries around the world. Puerto Rico is no exception. The investigation presented in this article will analyze the repercussions that the absence of protocols for the human trafficking of minors have wrought in the fight against this exploratory phenomenon in Puerto Rico. To achieve this purpose, an investigation was realized with a 
mixed focus and a exploratory design. The hypothesis of this study attempts to prove the negative effects of the lack of protocols in the fight against this phenomenon will be reduced with the presence of protocols against the human trafficking of minors in Puerto Rico. It was also auscultated if the identification and inclusion of the correct factors in a protocol for minors could improve the detection strategies in these cases. For this study, the knowledge of experts that offer services to minors in public agencies in Puerto Rico, as well as non-government agencies (NGO) was used. The selected sample was not randomly selected by availability. The technique utilized to obtain this information was the interview in the middle of a questionnaire. This questionnaire was redacted utilizing the Likert scale, alongside open questions. The main objectives to create a prototype plan of youth prevention for human trafficking and to identify the factors that a prevention and protection protocol for the human trafficking of minors must include in order to be effective were met.

Key words: human trafficking, human rights, minors, slavery and exploitation

\section{Introducción}

En todos los países del mundo existen diversidad de problemas sociales. Muchos de esos problemas dependen de diferentes factores como lo son: la pobreza, la cultura, la economía, las costumbres, tradiciones, condición política, social, localización geográfica y muchos otros. En los tiempos modernos, con la globalización, muchos de esos problemas pasan de un país a otro con más facilidad (Rey y Hernández, 2010). Lamentablemente el problema de la trata humana no es la excepción a esa realidad. La trata humana, también conocida como trata de personas y como la esclavitud moderna, es un problema social que puede trascender fronteras. La trata, puede crecer en las entrañas de cada país y viola los más elementales derechos humanos de las personas. En Puerto Rico, el problema de la trata es poco conocido y tanto al gobierno como a la sociedad, le cuesta aceptar su existencia (Rey y Hernández, 2010).

La trata humana fue tipificada como delito en el Código Penal de Puerto Rico, en el año 2012 (Ley Núm.146, 2012). La ausencia de protocolos de trata, dirigidos a la prevención y protección de los menores, unido a la escasez de estadísticas e información relevante con relación al tema y las limitadas investigaciones existentes en el país, despertaron el interés de la investigadora con relación a este tema. De esa inquietud, surgió la investigación que se presenta en este artículo.

En la actualidad la trata humana es considerada el segundo delito más lucrativo del mundo, después del tráfico de drogas (Fundación Ricky Martín, 2020). Se estima, que es un negocio que genera aproximadamente ciento cincuenta (150) mil millones de dólares en ganancias anuales en el mundo (Fundación Ricky Martín, 2020). En información publicada en Global Estimates of Modern Slavery (2017), de la International Labor Organización (ILO, por sus siglas en inglés), se hace un estimado de que, en el año 2016, se registraron 40.3 millones de víctimas de la esclavitud moderna a nivel mundial (ILO, 2017). Se cree que, de esa impresionante cifra, 5.5 millones, son menores de edad (Fundación Ricky Martin, 2020). En cuanto a las estadísticas de Puerto Rico se refiere, hasta el año (2018), no existían estadísticas de este delito (Senado de Puerto Rico, 2018). Asimismo, de acuerdo con el Centro de Estadísticas de la Policía de Puerto Rico (2020), hasta el 31 de julio del año 2019, se había acumulado un solo caso de trata humana (Policía de Puerto Rico, 2020).

De otra parte, este fenómeno se ha estudiado desde diferentes teorías, entre ellas, la teoría multifactorial. De acuerdo con esta teoría, las causas de la trata humana 
provienen de diferentes factores (Rey Hernández, 2010). Muchos de los defensores de esta teoría, entienden que la conducta criminal puede responder a una combinación de factores y circunstancias, tanto del sujeto como del ambiente que lo rodea (Rey y Hernández, 2010). Otra teoría que ha tomado pertinencia en el mundo moderno y que se está utilizando en diversas investigaciones relacionadas a este tema, es la teoría de la globalización. Aunque inicialmente se entendía que era un movimiento económico, esa facilidad del acercamiento entre naciones ha traído consecuencias en el ámbito social, político, cultural, tecnológico y en muchos otros aspectos. (Irwin N., 2016). Estas dos teorías, fueron la piedra angular de la investigación.

Por otro lado, aunque en Puerto Rico, existe una escasez de investigaciones relacionadas con el tema de la trata humana, y el tema es poco conocido, en los últimos años se ha comenzado a reconocer la presencia de este fenómeno. Esto ha ocurrido gracias a las investigaciones lideradas por la Fundación Ricky Martín, en colaboración con la Universidad de Puerto Rico y el Protection Project en la Escuela Avanzada de Estudios Internacionales de la Universidad de Johns Hopkins. De dicha fundación, han surgido tres importantes investigaciones relacionadas con este tema. Al presente, esos son los trabajos más importantes realizados sobre dicho tema en Puerto Rico. Fue con la primera investigación, que prácticamente salió a la luz pública la existencia del problema de la trata en Puerto Rico. En esa primera investigación, se identificaron los primeros tipos de trata existentes en la isla. Esa primera investigación, lleva por título: La trata de personas en Puerto Rico un reto a la invisibilidad (2010). Las tres investigaciones estuvieron a cargo de dos prestigiosos profesores de la Universidad de Puerto Rico, el Dr. César Rey y la Dra. Luisa Hernández. Con la primera investigación se destapa a nivel nacional, la existencia del problema de la trata y la posible magnitud de este fenómeno en Puerto Rico. En la segunda investigación, que lleva por título: La trata de personas: Una forma Moderna de esclavitud en Puerto Rico (2014), los investigadores descubrieron nuevos tipos de trata presentes en Puerto Rico. Además, tuvieron la oportunidad de entrevistar algunas víctimas de este delito. La tercera investigación, fue titulada Violencia género y trata (2017) y fue liderada por la Dra. Luisa Hernández. En la misma, se auscultó la relación entre las víctimas de violencia de género y la trata humana. Cabe señalar, que las investigaciones realizadas por la Fundación Ricky Martín y la Universidad de Puerto Rico han sido fundamentales para la realización del trabajo de investigación que se presenta en este artículo.

Para tener una mejor perspectiva del problema de la trata, es importante discutir de una manera un poco más amplia algunas de las investigaciones realizadas en Puerto Rico. Antes de abundar en esas investigaciones, es pertinente la definición de algunos términos que suelen ser confundidos, esos términos son:

Tráfico humano

De acuerdo con la definición de las Naciones Unidas (ONU), tráfico humano se refiere a:

Por tráfico ilícito de migrantes se entenderá la facilitación de la entrada ilegal de una persona en un Estado Parte del cual dicha persona no sea nacional o residente permanente con el fin de obtener, directa o indirectamente, un beneficio financiero $u$ otro beneficio de orden material (ONU, 2000, p.57).

Trata humana

Según la Organización de las Naciones Unidas (ONU), la definición de trata humana es: 
La captación, el transporte, la acogida o la recepción de personas, recurriendo a la amenaza o al uso de la fuerza u otras formas de coacción, al rapto, al fraude, al engaño, al abuso de poder o de una situación de vulnerabilidad o a la concesión o recepción de pagos o beneficios para obtener el consentimiento de una persona que tenga autoridad sobre otra con fines de explotación. Esa explotación incluye, como mínimo, la explotación de la prostitución ajena u otras formas de explotación sexual, los trabajos o servicios forzados, la esclavitud o las prácticas análogas de la esclavitud, la servidumbre o la extracción de órganos" (ONU, 2000, p. 44 y 45).

A pesar de la escasez de investigaciones sobre el tema en Puerto Rico, no se puede negar que, se han realizado estudios de los que se ha obtenido información relevante. Una de esas investigaciones lleva por título: Migración Clandestina: Informe preliminar sobre la Trata de Personas desde República Dominicana hacia Puerto Rico, realizada por la Organización de Estados Americano (OEA por sus siglas en inglés), realizada en el año 2006. Esta investigación, consta de más de diez años de realizada. Sin embargo, cobra vital importancia ya que aparenta ser la primera realizada por una organización internacional de prestigio, que identifica la existencia del problema de la trata en Puerto Rico. Posteriormente, Puerto Rico también fue incluido en el informe, Congresional Reseach Services (CRS, por sus siglas en inglés) Report for Congress (2013. Aparentemente es en el informe de ese año que por primera vez se identifica a Puerto Rico como un país de origen, tránsito y destino de la trata humana (Departamento de Estado de los Estados Unidos, 2013). En cuanto al estudio realizado por la OEA, se intenta analizar y entender la posibilidad de la existencia de la trata humana en Puerto Rico (OEA, 2006). En él informe, se expuso la existencia de un alto volumen de inmigrantes dominicanos que entraban a Puerto Rico. Lo que podía levantar la sospecha de que la isla, funcionara como un país de tránsito y destino para las víctimas de la trata (OEA, 2006).

De igual forma, otra investigación importante, que estudia este tema es la titulada: Trata de personas; una forma moderna de esclavitud en Puerto Rico, (Rey y Hernández, 2014). En dicha investigación, los autores tuvieron la oportunidad de entrevistar algunas víctimas de trata en Puerto Rico. Una de las historias más impactantes que surgió de dichas entrevistas, fue la de una menor de edad, que aseguró que su madre la prostituía desde que tenía cinco años. Según el relato de la menor, la madre la encerraba en una habitación junto a su hermana, las amarraba a la cama y cobraba la cantidad de cien dólares $(\$ 100.00)$ para que hombres sostuvieran relaciones sexuales con ellas (Rey Hernández, 2014). Algunos hogares sustitutos (entiéndase por estos, hogares bajo la supervisión del Departamento de la Familias de Puerto Rico, donde se ubican los menores removidos de sus hogares por maltrato $u$ otras acciones que puedan ponerlos en riesgo), también son focos de este fenómeno (Rey y Hernández, 2014). En algunos de estos hogares, los menores son víctimas de abuso sexual (Rey y Hernández, 2014). De acuerdo con los autores, la vulnerabilidad de los niños y niñas los hacen presa fácil de otros predadores (Rey y Hernández, 2014).

De otra parte, en el Informe de Trata de Personas CRS Report for Congress (2013), queda establecido que, Puerto Rico es utilizado como lugar de origen, tránsito y destino, para el delito de la trata (Departamento de Estado de los Estados Unidos, 2013). Con esa aseveración coinciden diferentes estudiosos del tema, como lo son: María Salado (2014), el Dr. César Rey y la Dra. Luisa Hernández (2010), entre otros. Asimismo, en el estudio Migración Clandestina: Informe preliminar sobre la Trata de Personas desde República Dominicana hacia Puerto Rico (2006), uno de los señalamientos más 
importantes, fue en el sentido de que tanto las autoridades de Estados Unidos, como las de Puerto Rico, tenían falta de comprensión sobre este asunto (OEA, 2006). Lo que llama la atención, es que, según investigaciones subsiguientes, como La Trata de Personas en Puerto Rico: un reto a la Invisibilidad (2014), se argumenta, que aún existe esa falta compresión sobre el problema de la trata humana en Puerto Rico (Rey y Hernández, 2014).

Otra investigación importante, sobre el tema de la trata en Puerto Rico, es la más reciente que realizara la Fundación Ricky Martín, también liderada por los profesores Luisa Hernández y César Rey. Dicha investigación lleva por título: Violencia: género y trata (2017). En dicha investigación, se ausculta la posibilidad de que dentro del grupo de mujeres que sufren violencia de género, también se encuentren mujeres víctimas de trata humana. Dicho estudio fue exploratorio y se realizó mediante una revisión documental de expedientes de mujeres que han buscado ayuda en la Oficina de la Procuraduría de la Mujer (OPM) del Estado Libre Asociado de Puerto Rico. Uno de los hallazgos más importantes de la investigación, fue descubrir que La Oficina de la Procuraduría de la Mujer, es un buen lugar para identificar víctimas de trata humana en Puerto Rico. Según los investigadores, sería importante adiestrar a estos fines, al personal de dicha procuraduría, particularmente a los que realizan la entrevista inicial (Hernández y Rey, 2017).

De acuerdo con el estudio, se estima que en Puerto Rico el cuarenta y seis puntos dos por ciento (46.2 \%) de las personas, viven bajo el nivel de pobreza. El sesenta y dos por ciento $(62 \%)$ de las mujeres viven en una situación aún peor, al igual que el sesenta y ocho por ciento (68\%) de sus hijos. (Pares Maga, 2016, citado por Rey y Hernández, 2017). Se entiende que uno de los sectores de mayor vulnerabilidad, son los hogares donde las mujeres son las jefas de familia. (Rey y Hernández, 2017). Dentro de ese grupo, también se identifican mujeres migrantes que acuden a la OPM a buscar ayuda. Cabe señalar, que la vulnerabilidad, es una de las características de las víctimas de trata humana. De acuerdo con los investigadores, muchas de las mujeres migrantes, ejercían labores de cuidadoras y empleadas domésticas. Situación que, sin duda alguna las podía colocar en una posición vulnerable para convertirse en potenciales víctimas de trata humana y explotación. Lograr la confirmación de que las víctimas de violencia de género son sumamente vulnerables al tráfico y a la trata, fue uno de los hallazgos más destacados y relacionados al propósito de la investigación (Hernández y Rey, 2017).

Por otro lado, no se puede hablar del tema de la trata, sin estudiar las legislaciones, tratados y protocolos internacionales y nacionales con relación a este tema. A continuación, se mencionan algunos de ellos:

En cuanto a la legislación y protocolos se refiere, el Protocolo para prevenir, reprimir y sancionar la trata de personas especialmente mujeres y niños, que complementa la Convención de las Naciones Unidas contra la Delincuencia Organizada Transnacional, mejor conocido como Protocolo de Palermo (2000), en la actualidad es considerado como el instrumento internacional más importante en la lucha contra la trata de personas. Fue firmado por los Estados en el año 2000 (entiéndase por Estados, los países que han ratificado alguno de los protocolos de la Organización de las Naciones Unidas). Entró en vigor en el año 2003 y fue ratificado por 124 países en el año 2009. Es prácticamente la guía que utilizan todos los Estados firmantes del protocolo de Palermo, 
para redactar y trazar sus estrategias de lucha contra este fenómeno. También, existe la Ley Modelo Contra la Trata de Personas (2010), la misma fue creada por la Oficina de las Naciones Unidas contra la Droga y el Delito (UNODC, por sus siglas en inglés). Dicho modelo de ley se crea con el propósito de sistematizar la prestación de asistencia legislativa por la UNODC y facilitar la legislación de los Estados firmantes del Protocolo de Palermo. Está diseñada para adaptarse a la legislación de cualquiera de los Estados, sin importar tradición jurídica y sus condiciones sociales, económicas, culturales y geográficas (UNODC, 2010). La denominada Ley Modelo, es una guía para que los Estados firmantes del Protocolo de Palermo, la puedan adaptar a sus culturas y necesidades. De tal modo, que se mantenga uniformidad entre la legislación de estos, con lo acordado y firmado en el Protocolo de Palermo (UNODC,2010).

Han sido muchos los protocolos y guías que se han desarrollado alrededor del mundo para combatir este fenómeno. Aparte del Protocolo de Palermo, también se encuentran el Protocolo Facultativo de la Convención sobre los Derechos del Niño relativo a la venta de niños, la prostitución infantil y la utilización de niños en la pornografía (2000). De igual forma, se creó El Protocolo facultativo de la Convención sobre los Derechos del Niño relativo a la participación de niños en los conflictos armados (2000) y muchos otros.

En algunos países se ha legislado y tipificado como delito la trata de personas. A continuación, se mencionan algunas de esas leyes: ley 26842 trata de personas y asistencia a sus víctimas. Prevención y Sanción. Código Procesal Penal, modificado el 19 de diciembre de 2012, en Argentina, ley 896 Ley Contra la Trata de Personas, aprobada el 28 de enero de 2015 en Nicaragua, ley DOF 19-03-2014, ley General Para Prevenir, Sancionar y Erradicar los Delitos en Materia de Trata de Personas y para la Protección y Asistencia a las Víctimas de Estos Delitos, Estados Unidos Mexicanos, ley 9095 Ley Contra la Trata de Personas, publicada el 8 de febrero 2013, en Costa Rica, entre muchas otras.

En Estados Unidos y Puerto Rico también se han aprobado legislaciones dirigidas a combatir la trata. Entre las leyes aprobadas por los Estados Unidos se encuentran: Ley Pública 106-386 Ley de Protección a Víctimas de Trata de Personas - octubre (2000) (TVPA, por sus siglas en inglés). Esta ley fue creada con el propósito de asegurar un castigo justo y eficaz a los traficantes y para la protección de las víctimas. Es la primera ley federal de los Estados Unidos que cubre tres áreas importantes como lo son: prevención (prevención), protección (protección) y enjuiciamiento (prosecución), mejor conocido como la tres (3) P. La TVPA, estableció varios métodos encaminados a enjuiciar los traficantes, prevenir la trata de personas y ofrecer protección a las víctimas. Clasifica la trata de personas como delitos federales e impone severas penas a los traficantes. Además, crea la Oficina de Monitoreo y Combate a la Trata de Personas, como una forma de prevenir la trata. Dicha oficina, debe monitorear el trabajo que hacen los diferentes países, para cumplir con los estándares mínimos establecidos por la TVPA (2000). Esa encomienda, se realizará mediante la publicación de un Informe de Trata de Personas (TIP, por sus siglas en inglés). Dicho informe, evalúa y describe los esfuerzos de los países, para luchar contra la trata humana (TVPA, 2000). Además, la ley (TVPA) estableció la Fuerza de Tarea Interinstitucional Para Monitorear y Combatir la trata y la implementación de la ley. En adición, la ley protege a las víctimas y sobrevivientes de la trata mediante el establecimiento de lo que se conoce como la Visa T. Esa visa, permite que las víctimas de trata y sus familiares cercanos, permanezcan en los Estados Unidos como residentes temporales y les ofrece la opción de convertirse en residentes permanentes, al cabo de tres (3) años (TVPA, 2000). Además, cuentan con la Ley de 
Reautorización de la Protección de Víctimas de la Trata (TVPRA, 2003). Esta ley estableció un derecho de acción federal y civil para que las víctimas de la trata puedan demandar a los traficantes (TVPA, 2003, Sec. 1595). Además, incluyó el delito de la trata a los delitos que se pueden considerar bajo el estatuto de Organizaciones Corruptas Influenciadas por el Delito de Corrupción (RICO, por sus siglas en inglés). En adición a esas medidas, incluyó medidas de protección a las víctimas y sus familiares con relación a la deportación y también incluyó un requisito, con relación a que el Fiscal General debe realizar un informe anual sobre los esfuerzos realizados por los países para combatir la trata. Ese informe debe ser presentado al Congreso. (TVPRA, 2003) Además, cuentan con la Ley de Reautorización de la Protección de Victimas de la Trata (TVPRA, 2013), la misma fue aprobada como enmienda a la Ley contra la Violencia contra la Mujer. Esa ley refuerza programas que garanticen que los ciudadanos no consuman productos fabricados por víctimas de la trata y para prevenir los matrimonios. Incluye también, respuestas de emergencia en el Departamento de Estado para que haya una respuesta rápida, cuando surjan casos de desastres naturales. Situación que aumenta la vulnerabilidad de las personas a ser víctimas de trata. También, refuerza la colaboración con las fuerzas de ley y orden locales y estatales para viabilizar y agilizar el enjuiciamiento de los tratantes (TVPRA, 2013), entre otras muchas legislaciones aprobadas.

Existe legislación federal de los Estados Unidos de América, que es aplicable a Puerto Rico. Ello se debe a la relación política que existe entre ambos países. Sin embargo, ambas culturas tienen grandes diferencias, por cuanto el pueblo de Puerto Rico se ve en la necesidad de presentar legislación atemperada a su idiosincrasia y realidad como pueblo. Atendiendo las necesidades del pueblo puertorriqueño, la trata humana queda tipificada como delito en el artículo ciento sesenta (160) del nuevo Código Penal (2012). La trata fue ubicada como parte de los delitos contra los derechos civiles de los ciudadanos puertorriqueños. En el nuevo Código Penal de Puerto Rico, la trata quedó definida y tipificada como una conducta criminal de la siguiente manera:

El que una persona mediante la captación, el transporte, el traslado, la acogida o recepción de personas, y que recurriendo al uso de la fuerza, amenaza, coacción, secuestro, fraude, engaño, abuso de poder, u otras situaciones de vulnerabilidad, ofrezca o reciba la concesión o recepción de pagos o beneficios con el fin de obtener el consentimiento de una persona que tenga autoridad sobre otra para que esta ejerza la mendicidad, cualquier clase de explotación sexual, pornografía, trabajo o servicio forzado, servidumbre por deudas, matrimonio servil, adopción irregular, esclavitud o sus prácticas análogas, la servidumbre o extracción de órganos, aún con el consentimiento de la víctima (Ley Núm. 146-2012, p.78).

En el mencionado artículo, también se llama la atención a diferentes modalidades de explotación. En específico la explotación sexual, trabajos forzosos, servidumbre por deudas, matrimonio servil, adopción irregular, esclavitud, servidumbre y la extracción de órganos. La servidumbre involuntaria, quedó definida en otro artículo del Código Penal, aunque también está incluido en el artículo 160 de trata humana (Ley 146-2012). Además, bajo el Artículo 300, del mismo Código Penal (2012), bajo crímenes de lesa humanidad, queda incluida la trata humana, según se definida en el artículo 160 del Código Penal de Puerto Rico (Ley 146-2012).

De igual forma, se enmendaron otras leyes para incluir la trata como delito, este fue el caso de la ley de menores Ley Núm. 225 de 2014, para enmendar los artículos 2, 3, 5, 7, 8, 11 y 58 de la Ley Núm. 246, Ley Para la Seguridad, Bienestar y Protección de 
Menores (2014), en la cual se incluye la trata humana como una de las formas de maltrato. Igualmente, se aprobó la Ley Núm. 87 de 2015, en la que se declara el mes de febrero como Mes de Orientación y Prevención Contra la Trata Humana en Puerto Rico, incluyendo la celebración del Día de la Orientación y Prevención Contra la Trata Humana, el 3er lunes de febrero de cada año, con el fin de concienciar a los habitantes de Puerto Rico, sobre su existencia, efectos y formas de contribuir a su erradicación; y para otros fines (Ley Núm.87-2015 p.1).

\section{Método}

Con el propósito de estudiar las consecuencias que acarrea la ausencia de protocolos, para la prevención y protección de la trata humana en menores de edad, y el posible efecto que esto ha ocasionado en la lucha contra este fenómeno en Puerto Rico, surgió la presente investigación. El estudio, se realizó utilizando una metodología con un enfoque mixto, que implica un proceso de recolección, análisis y vinculación de datos de ambas metodologías, cuantitativa y cualitativa (Hernández, Sampieri et al., 2014). Lo que ofrece a la investigación unos resultados más completos y una forma más amplia de estudiar un problema tan complejo y desconocido en Puerto Rico. Según Hernández Sampieri (2014), las investigaciones mixtas, generan inferencias cualitativas y cuantitativas, lo que se conoce como meta-inferencias. Además, menciona como algunos de sus beneficios, los mencionados a continuación: perspectiva más amplia y profunda, datos más ricos y variados, mayor solidez y rigor y otros (Hernández Sampieri, 2014). Con esa postura coinciden Ugalde y Balbastre (2013), que son los autores del artículo Investigación Cuantitativa e Investigación Cualitativa: Buscando las Ventajas de las Diferencias Metodológicas de Investigación (2013). Dichos autores, exponen que las investigaciones mixtas les ofrecen a los resultados una mayor confiabilidad, validez y comprensión.

En la investigación mixta se integran ambos enfoques (cuantitativo y cualitativo). Esa integración, ofrece la oportunidad de unos resultados más completos (Ugalde y Balbastre, 2013). La obtención de resultados con más fuerza y el que se contrarresten las desventajas de las metodologías cualitativas y cuantitativas (cuando son utilizadas de forma individual), son otras de las ventajas de utilizar dicho enfoque (Ugalde y Balbastre, 2013). Este enfoque, ofreció la oportunidad de realizar una investigación con un punto de vista más amplio.

De igual forma, utilizar este método de investigación, brindó la oportunidad de obtener una visión más amplia y completa del fenómeno de la trata humana en Puerto Rico. Con la utilización de este método, se persiguió hacer uso de lo mejor de cada uno de los enfoques, entiéndase cualitativo y cuantitativo. Con ello se intentó reducir las posibles debilidades que pudiera tener cada uno de los enfoques. Utilizar este método, ofreció la conveniencia de indagar en el tema en cuestión de una manera más abarcadora.

En cuanto al diseño se refiere, en la investigación se utilizó el diseño exploratorio. Como se ha expuesto anteriormente, en Puerto Rico, la trata humana es un tema relativamente nuevo y hay escasez de investigaciones con relación al mismo. (Rey y Hernández, 2014). No se puede perder de perspectiva, que la investigación con diseño exploratorio se utiliza para aumentar el grado de conocimiento del tema a ser estudiado (López, 2014). Además, le brindó a la investigación una perspectiva innovadora. El uso de este diseño ofrece la oportunidad de que, a partir de los resultados de la investigación, 
surja el interés de nuevas investigaciones (López, 2014). Lo que hizo este diseño, el ideal para la investigación del tema de la trata en Puerto Rico.

Asimismo, los objetivos más importantes trazados para el estudio fueron: identificar los factores que debía incluir un prototipo de protocolo de trata humana para menores de edad, desarrollar un prototipo de plan de prevención juvenil de trata humana, adaptado a la realidad puertorriqueña y otros. Los factores, fueron identificados por los participantes del estudio.

Con relación a las hipótesis, buscaban establecer que efectivamente existe una relación entre la presencia de protocolos de prevención de trata para menores, y la posible reducción en los efectos que ha tenido la ausencia de estos, en la lucha contra este fenómeno en Puerto Rico. Además, se auscultó la posibilidad de que al identificar los factores correctos que debe incluir un protocolo de prevención de trata humana para menores, se podía mejorar la detección de casos en Puerto Rico.

Para realizar la investigación, se utilizó una muestra no aleatoria y por disposición. La muestra se obtuvo de las agencias pertenecientes al sistema de justicia criminal, la agencia encargada de velar por el mejor bienestar de los menores en Puerto Rico que se conoce como Departamento de la Familia y dos ONG. Las agencias invitadas a participar fueron:

1. Departamento de la Familia (Administración de familias y niños),

2. Instituciones juveniles del Departamento de Corrección y Rehabilitación

3. Policía de Puerto Rico

4. Rama judicial

5. Departamento de Justicia

6. ONG, se contó con la colaboración de personal de los Hogares CREA y de Teen Challenge, ya que estás organizaciones ofrecen servicios a jóvenes menores.

De otra parte, la construcción del instrumento se dirigió a auscultar el conocimiento y la percepción de los sujetos, sobre el tema en cuestión. La validación del instrumento estuvo a cargo de tres jueces profesores con título doctoral de una importante universidad en Puerto Rico, que, a su vez, son conocedores de la metodología de la investigación. Se espera que la información recopilada mediante el instrumento de la investigación ofrezca a las agencias gubernamentales y ONG participantes del estudio, información relevante con relación al tema de la trata en Puerto Rico.

Así mismo, el instrumento cumplió con su propósito de obtener la información necesaria para realizar la investigación. La muestra, se obtuvo de forma no aleatoria y por disposición. Se espera que los resultados obtenidos de la investigación hagan del estudio una aportación para futuras investigaciones. Como ha quedado establecido, en Puerto Rico existe una escasez de investigaciones en cuanto al tema en cuestión.

Referente a las limitaciones del estudio, las más importantes fueron: la escasez de investigaciones e información, relacionadas con el tema en Puerto Rico, el temor de algunos funcionarios gubernamentales a responder preguntas que entendían que evaluaban el sistema para el cual trabajan, los requisitos para ofrecer el permiso para realizar la investigación en algunas agencias, el paso del Huracán María, la escasez de información con relación al tema de la trata humana en Puerto Rico y otras. Es importante señalar que en una de las agencias no se consiguió de forma oficial la autorización para 
llevar a cabo el estudio, ya que tenían exigencias que atrasarían la realización de la investigación. De igual forma, se consiguió entrevistar representantes de la agencia que consintieron sin problema alguno a participar de la investigación. En otra de las agencias, aunque se consiguió la autorización, se hizo imposible la comunicación para coordinar la realización de las entrevistas, sin embargo, se logró contactar algunos empleados que accedieron a participar de la investigación. Esta investigación es una aportación adicional a los estudios que ya existen sobre el tema de la trata en Puerto Rico, lo cual puede beneficiar a futuros investigadores de este asunto. De la investigación, pueden surgir preguntas adicionales que abran el espacio para nuevos estudios.

En cuanto a los hallazgos de la investigación, uno de los más significativos fue encontrar que, un importante número de las agencias gubernamentales participantes que ofrecen servicios a menores de edad en Puerto Rico, no están debidamente preparadas para detectar y ofrecer servicios a menore que son víctimas de trata humana. Por lo que, las víctimas pueden estar siendo ignoradas por el estado o victimizadas doblemente, en primer lugar, por sus captores y en segundo lugar por el estado. Además, se encontró que muchos de los empleados expresaron no sentirse preparados para detectar ni ofrecer servicios a menores víctimas o posibles víctimas de este fenómeno. En su mayoría los entrevistados demostraron tener por lo menos un conocimiento básico de lo que es la trata y de su existencia en Puerto Rico. Sin embargo, no se puede olvidar que estás son las personas llamadas para ofrecer los servicios a esa población tan vulnerable. Otro hallazgo importante, fue la ausencia o escasas estadísticas sobre el delito de la trata en Puerto Rico, aun cuando desde el año 2012, la trata fue tipificada como delito en Código Penal de Puerto Rico. Además, un grupo importante de los entrevistados, indicaron no conocer ni el Protocolo de Palermo ni algunas de las leyes relacionadas a la trata humana, que son aplicables en Puerto Rico. Otro significativo hallazgo que surgió de la investigación fue que tanto el Departamento de la Familia como la Policía de Puerto Rico, cuentan con protocolos de intervención para casos de trata humana. Sin embargo, de la investigación no se encontró evidencia de la existencia de protocolos nacionales de prevención de trata humana para menores de edad. Estos hallazgos y muchos otros, surgidos de la investigación, evidencian la necesidad de constante capacitación y orientación a los empleados de primera fila en ofrecer servicios a menores de edad. No se puede obviar que la trata humana, todavía al presente, es un tema poco conocido y estudiado en Puerto Rico.

\section{Resultados}

Dentro de los resultados más significativos de la investigación, se puede mencionar que los entrevistados identificaron los factores que debe incluir un prototipo de plan de prevención juvenil. Con la información ofrecida por los entrevistados, se cumplió con el objetivo principal del estudio, el cual consistía en crear un prototipo de plan de prevención juvenil de trata humana. Además, los entrevistados identificaron los factores que deben estar incluidos en un prototipo de protocolo de prevención y protección de trata humana para menores de edad puertorriqueños. También, se pudo constatar la inseguridad de algunos de los empleados para manejar casos de trata humana. Se pudo identificar el deseo genuino de muchos de los participantes, en cuanto a estar capacitados para identificar, atender y prevenir este tipo de situaciones.

De la investigación resultaron evidentes, la necesidad de protocolos estatales y de vanguardia para la prevención y protección de trata humana para menores de edad. 
Asimismo, quedó en evidencia la importancia y necesidad de la capacitación, orientación, concienciación y responsabilidad con relación a este fenómeno. Los entrevistados fueron enfáticos, en cuanto a la importancia de ofrecerle prioridad a estos asuntos. Los participantes sugirieron, que se creen protocolos que estén acompañados de directrices claras que ayuden a los empleados a identificar los posibles casos de trata., ya que algunos expresaron no sentirse capacitados para ello. Además, quedó implícita la falta de comunicación entre las agencias pertinentes, la falta de definición de procedimientos y la necesidad de una definición más clara y comprensible del significado de la trata. Lo que devela la necesidad de un protocolo nacional de prevención y protección de trata humana para menores de edad. Ese protocolo, deberá estar acompañado por una guía que contenga directrices específicas y claras en los casos de detección de casos de trata. Asimismo, se expresó la urgencia de definir las responsabilidades de cada agencia pertinente en este asunto. El protocolo debe verse como una herramienta adicional que ayude en la lucha contra este fenómeno en Puerto Rico. Dicho protocolo debe servir de guía para los empleados de agencias públicas y de ONG en Puerto Rico.

De igual forma, los participantes del estudio identificaron algunos de los efectos que ha tenido la ausencia de protocolos, en la lucha contra este fenómeno en Puerto Rico. De acuerdo con el conocimiento y percepción de los entrevistados, algunos de esos efectos son: atrasos en los procedimientos criminales en las agencias del Sistema de Justicia Criminal, incapacidad de algunos funcionarios para identificar actos de trata humana, mayor vulnerabilidad para que los menores sufran actos de violencia, mayor exposición a la violación de los derechos humanos de las posibles víctimas, ausencia de herramientas de intervención de parte de los funcionarios, un atraso en la radicación de casos y otros.

Por otro lado, otro importante resultado identificado por medio de la investigación fue, la necesidad de establecer indicadores que sirvan de guía para reconocer posibles casos de trata, la necesidad de capacitación para los funcionarios que ofrecen servicios a esta población, definición de las obligaciones de cada agencia y directrices específicas a cada una, en cuanto a cómo proceder al detectar casos de trata. También, se identificó la importancia de promover la buena comunicación entre las agencias con el propósito de lograr un buen trabajo en equipo, una buena comunicación, coordinación y agilización de los servicios a ofrecer, lo que debe redundar en beneficio para los menores.

\section{Discusión y conclusiones}

Conforme a los resultados de la investigación, se hace inminente contar con un protocolo estatal de prevención y protección de trata humana para menores en Puerto Rico. Además, es indispensable educar y capacitar a los funcionarios públicos, a los funcionarios de ONG y a la comunidad en general, sobre este tema y su magnitud. Es imperativo una divulgación masiva y constante de la realidad sobre la existencia de este fenómeno en la isla. Según los resultados del estudio, es necesario crear alianzas públicoprivadas para combatir de forma efectiva este problema. Queda clara, además, la necesidad de alianzas con otros países, sobre todo, países latinoamericanos también afectados por este asunto. Para trabajar en conjunto y coordinación en la lucha contra es fenómeno.

A pesar de que Puerto Rico se ha reconocido, como un país de origen, tránsito y destino de casos de la trata humana, todavía al presente, existe mucho desconocimiento sobre este tema. Sin embargo, los entrevistados mostraron mucho interés y preocupación por estar capacitados para identificar y ofrecer los servicios adecuados a las víctimas de 
este delito. En algunos funcionarios se pudo percibir un tono de frustración y preocupación con relación a este asunto.

No se puede negar que el gobierno de Puerto Rico ha tenido avances en cuanto a legislación nacional se refiere. Sin embargo, ha quedado clara la necesidad de trabajar con la prevención y protección de los menores. Todavía el problema de la trata no es completamente visible en la comunidad puertorriqueña. Evidentemente falta un largo camino que recorrer en cuanto a este asunto se refiere. Se hace urgente el manejo adecuado de este fenómeno.

Es fundamental involucrar a la comunidad como parte integral de la lucha contra este problema. La educación, en todos los niveles, es una herramienta indiscutible para enfrentar este monstruo. Este fenómeno, amenaza la seguridad y bienestar, no solo de los niños y niñas puertorriqueños, si no, de los niños y niñas del mundo entero. No se puede escatimar en esfuerzos cuando se trata del mejor bienestar de los menores. A veces, parece que se olvida que los menores son el futuro de los pueblos.

Queda en manos de los miembros de la rama legislativa, la rama ejecutiva y de la rama judicial de Puerto Rico, prestar la atención que merece este asunto. Recae sobre sus hombros, la responsabilidad de proveer las herramientas necesarias para minimizar los efectos que ocasiona la ausencia de protocolos de trata humana para menores de edad, en la lucha contra este fenómeno. Además, son ellos los responsables de velar por los mejores intereses de los menores puertorriqueños.

\section{Referencias}

Binda, N. U., \& Balbastre-Benavent, F. (2013). Investigación cuantitativa e investigación cualitativa: buscando las ventajas de las diferentes metodologías de investigación. Revista de Ciencias económicas, 31(2), 179-187.

Hernández L., Rey C. (2017), Violencia: género y trata. Puerto Rico: UPR

Hernández Sampieri R.; Fernández-Collado, C. \& Baptista Lucio, P. (1997 y 2010), Metodología de la investigación. México: McGRAW-HILL

(ILO), I. L. (2017). Global Estimates of Modern Slavary. Geneva Retrieved from https://www.ilo.org/global/publications/books/WCMS 575479/lang-en/index.htm

Irwin N, (2016) How Brexit Will Affect the Global Economy, Now and Later, New York Times. Retrieved from http://www.nytimes.com/2016/06/25/upshot/how-brexitwill-affect-the-global-economy-now-and-later.html?_r=0

Law 106-386. (2000). Victims of Trafficking and Violence Protection Act of 2000. E.U. Retrieved from https://www.govinfo.gov/app/details/PLAW-106publ386

Ley 225, Ley para enmendar los artículos 2,3,7,8,11 y 58 de la Ley Núm.246, Ley Para la Seguridad, Bienestar y Protección de Menores. (2014). Puerto Rico.

Ley 26842 de Asistencia a sus víctimas, prevención y sanción Código procesal penal Victims of Modificación.(2012).Argentina. Retrieved from https://www.argentina.gob.ar/normativa/nacional/ley-26842-206554 
Ley 87, Ley de Asistencia a Inmigrantes Víctimas de Trata Humana. (2015). Puerto Rico.

Ley 896, Ley contra la trata de personas. (2015, January 28). Nicaraguas. Retrieved from https://oig.cepal.org/sites/default/files/2015 nic ley896.pdf

Ley 9095, Ley contra la trata de personas. (2013). Ley contra la trata de personas. Costa Rica. Retrieved from https://oig.cepal.org/sites/default/files/2012 cri ley9095 0.pdf

Ley general para prevenir, sancionar y erradicar los delitos en materia de trata de personas y para la protección y asistencia a las víctimas de estos delitos (DOF). (2014, March 18). Estados Unidos Mexicanos. Retrieved from http://www.diputados.gob.mx/LeyesBiblio/pdf/LGPSEDMTP 190118.pdf

Ley Núm. (2012).146, Código Penal de Puerto Rico.

López W. (2014). Ocho pasos para el desarrollo de una investigación. Puerto Rico: UPR Apa, N. (2017). Normas Apa. Retrieved from Normas Apa: http://normasapa. net/2017edicion-6.

(ONU), O. d. (2000). Protocolo para revenir reprimir y sancionar la trata de personas, especialmente mujeres y niños, que complementa la Convención de las Naciones Unidas contra la Delincuencia OrganizadaTransnacional.

Rey C. y Hernández L. (2014). La trata de personas: Una forma moderna de esclavitud en Puerto Rico. San Juan, Puerto Rico: UPR

Rey C., Hernández. L. (2010). La trata humana en Puerto Rico; Un reto a la invisibilidad. San Juan, Perto Rico: UPR.

Salado, María (2014). La Trata de niños y niñas en Puerto Rico. Retrieved from https://prezi.com/asn0h_zlh-fs/la-trata-de-ninos-y-ninas-en-puerto-rico/

TVPRA, L. (2003). Ley de Reautorización de la Protección deVvíctimas de la Trata de Personas. Estados Unidos (E.U).

TVPRA, L. (2005). Ley de Reautorización de Protección de Víctimas de la Trata de Personas. Estados Unidos (E.U.).

TVPRA, L. (2008). Ley de Reautorización de Protección de Víctimas de la Trata de Personas. Estados Unidos (E.U).

TVPRA, L. (2013). Ley de Reautorización de la Protección de Víctimas de la Trata Estados Unidos (E.U.). 
Fecha de recepción: 05/05/2020

Fecha de revisión: 20/05/2020

Fecha de aceptación: 22/06/2020 